\title{
Reflexiones acerca del desarrollo emocional de la madre, a partir de la obra de Winnicott
}

Natalia Torres Vilar

En el trabajo se plantean algunas reflexiones en torno a la vivencia de la madre en el vínculo inicial con su bebé. Con este propósito se realiza, en primer lugar, una revisión general y sintética de lo que sería para Winnicott el desarrollo emocional del bebé, haciendo hincapié en el proceso que lleva a este desde los primeros esbozos del yo hasta la posibilidad del acto creativo. Esta revisión es luego complementada a la luz de la perspectiva del otro componente del vínculo: la madre. Se sugiere que junto con el desarrollo emocional del bebé, en casos normales, y a través de los fenómenos de empatía y de identificación cruzada, se da un reforzamiento del desarrollo emocional de la madre.

\section{Creatividad / empatía / identificación primaria / madre / self}

Reflections on the emotional development of the mother, on the basis of Winnicott's theory

This paper offers some insights into the experiences of mothers in their initial bonding with their children. To this end, the author offers a discussion of Winnicott's view of the emotional development of the baby, emphasizing the process from the first drafts of the ego to the possibility of the creative act. This discussion is enriched by the addition of the other component of the bond: the mother. It is suggested that, in normal cases, together with the emotional development of the baby and through the phenomena of empathy and crossidentification, a reinforcement of the emotional development of the mother takes place.

Creativity / empathy / primary identification / mother / self

Correo electrónico: ntorres@correo.ulima.edu.pe 
Para Winnicott el bebé y la madre son uno al comienzo. La madre "padece de un replegamiento" (1992a), centra su existencia en su hijo recién nacido, adaptándose a él. Este vínculo le permite captar en forma directa todo lo que sucede dentro de su hijo para poder satisfacer las necesidades de este. Si bien Winnicott planteó la relación madre-bebé como una unidad inseparable, afirmando que no existe tal cosa como un bebé, también podríamos decir que no hay tal cosa como una madre; la dupla es indisoluble. Sin embargo, la teorización de Winnicott (1979, 1990, 1992a, 1992b, 1993) se centró, según su interés, en el desarrollo emocional del infante. Lo que se desea resaltar en este trabajo es el otro polo de la unidad. Para ello intentaré plantear algunas reflexiones en torno a la vivencia de la madre en el vínculo inicial con su bebé. Abordaré, entonces, lo que he querido llamar un 'segundo proceso maduracional' de la madre, en el cual esta reeditará aspectos de su propio desarrollo emocional como infante -pero en circunstancias diferentes-, lo cual implicaría una crisis evolutiva que, como toda crisis, es una oportunidad de crecimiento, de elaboración; un nuevo nacimiento; la oportunidad para el surgimiento de un sí mismo más auténtico, para parafrasear a Winnicott.

Grete Bibring propuso esto ya en 1961. Para ella el embarazo constituye una crisis de maduración conducente a una nueva posición, no idéntica a la an- terior. En tanto crisis de maduración, el embarazo manifiesta regresión, distensión de las defensas y modificación en la organización del concepto del sí mismo. Pines (1972) enfatiza que, sobre todo en el primer embarazo, la tarea de separación-individuación se somete a una nueva evaluación y se completa. Joan Raphael-Leff (1995) explica las identificaciones complejas que se viven durante el embarazo, durante el cual la gestante -siendo una con su bebé- revive sus propias experiencias como feto, a la vez que se identifica con su propia madre para cumplir con su nuevo rol. Para esta autora, dichas identificaciones implican la modificación del yo en función de otro objeto (modificación que puede ser estable o transitoria e instrumental). Tomando estas ideas, las extiendo más allá del embarazo, como una vivencia paralela al desarrollo emocional del bebé en la infancia temprana.

Empezaré haciendo una revisión general y sintética de algunos aspectos de lo que sería el desarrollo emocional del bebé para Winnicott, para comentarla luego a la luz de la perspectiva del otro componente del vínculo: la madre.

Nos dice Winnicott (1979) que la vida psicológica comienza cuando aparece el yo, el primer esbozo de autoconciencia, la capacidad de percatarse del hecho simple de estar vivo, hecho expresado en la continuidad existencial, el registro del ser en el tiempo, en la captación elemental de la duración y 
en la realización y registro de los primeros gestos creativos, actos espontáneos capaces de inaugurar una existencia verdadera $\mathrm{y}$, por ende, dar nacimiento a un sí mismo verdadero.

En el inicio, nos dirá, el bebé y la madre son uno (Winnicott, 1992a). La madre experimenta una preocupación materna primaria, que se desarrolla gradualmente y se convierte en un estado de elevada sensibilidad durante el embarazo y especialmente hacia el final de este, y continúa hasta unas pocas semanas después del nacimiento del bebé. La madre "padece de un replegamiento", centra su existencia en su hijo recién nacido. Este es el vínculo que Winnicott llamó de identificación primaria o identidad. Este vínculo le permite captar, en forma directa, todo lo que sucede dentro de su hijo para poder satisfacer sus necesidades yoicas y pulsionales. Este replegamiento o disociación, llega a ser casi una enfermedad, de la cual luego la madre se recobrará. Sin embargo, es necesario que la madre conserve una pequeña parte de sí misma en contacto con la realidad externa, por lo que su estado ha sido considerado por Winnicott como una enfermedad esquizoide normal. Winnicott nos dirá también que esta preocupación primaria materna y este replegamiento no son fácilmente recordados por las madres una vez que se han recuperado de ellos, que tenderán a ser reprimidos.

Entonces, para Winnicott, un instrumento primordial en el desarrollo del bebé es la identificación, por la cual nos transportamos al interior de un objeto para coincidir con lo que tiene de único, y por consiguiente de inexpresable. Pero la identificación que permite la coincidencia con el objeto mismo se da en raras ocasiones. Quizá podríamos decir que para Winnicott la vivencia compartida por la madre y su bebé en los primeros momentos de la vida, es una de esas raras ocasiones. Esta identificación -no solo normal en el desarrollo del infante, sino también esencial para su supervivencia- solo puede ser vivida así posteriormente, en profundas regresiones psicóticas, y es básicamente incomunicable, al igual que el fenómeno en el vínculo madreinfante también se instala regresivamente para la madre en un momento previo a lo verbal.

Winnicott habla también de la creatividad primaria, que tiene que ver con que el pecho de la madre es constantemente recreado por el niño en virtud de su necesidad. La madre sitúa el pecho real en el lugar mismo en que el niño está dispuesto a crearlo, y en el momento adecuado. La ilusión tiene que ver con la omnipotencia en el niño, la idea de haber creado el objeto que encuentra. La madre se presenta como objeto para el bebé, a la vez que paradójicamente le deja creer que ella es su propia creación. El primer acto creativo, entonces, está en la posibilidad de crear un objeto que en la realidad se halla allí porque la madre, adaptada a 
las necesidades del yo de su bebé, lo colocó allí en el momento preciso y forma en que el bebé lo estaba creando.

Cuando la adaptación de la madre es adecuada, se produce en el bebé la ilusión de que la realidad corresponde a su capacidad para crearla. La madre suficientemente buena para Winnicott vendría a ser aquella que se adapta a las necesidades de su hijo, de manera adecuada al momento madurativo. Al inicio la adaptación es tal que el bebé no percibe esto, solo se percata del ambiente protector cuando siente sus fallas, debido a la ruptura de la continuidad existencial que estas provocan. $\mathrm{La}$ adaptación al medio incluye necesariamente la posibilidad de la falla. El bebé aprende a tolerar la ausencia gradualmente, en base a la confianza de la reaparición de la madre. Ella instaura el proceso de desilusión, sobre la ilusión que había ayudado a crear previamente. La madre no es capaz de aportar en forma ininterrumpida todo lo que su hijo necesita, y entonces el bebé empieza a registrar las ausencias y las frustraciones, poniéndose en cuestión su vivencia omnipotente e iniciándose el reconocimiento del mundo exterior. Sin embargo, la falla precoz y persistente de la madre, si esta le impone la alteralidad y no permite que el bebé la descubra, obliga al infante a adaptarse al medio y a convertirse en madre de sí mismo para sobrevivir. La madre suficientemente buena es una madre ideal, capaz de hacer experimentar al niño la frustración necesaria para el desarrollo de su deseo y su capacidad de individuación.

La omnipotencia del bebé implica que él cree que otras personas responden mágicamente a sus necesidades, apareciendo cuando él las convoca. Poco a poco entenderá que hay objetos que están fuera de su control. La desilusión gradual sólo es posible si se tuvo suficiente oportunidad para la ilusión. De lo contrario el bebé se resiste a abandonar el pensamiento mágico para no aceptar la frustración con la consiguiente pérdida de capacidad para reconocer la realidad. Allí se instauraría la omnipotencia como patología. Solo cuando existe confianza en el ambiente es posible el despliegue de la ilusión. La madre debe aceptar en principio la omnipotencia de su bebé, y ofrecerle la ilusión de un mundo creado por él. Poco a poco, en forma gradual, lo va desilusionando, con el fin de permitirle reconocer la realidad.

Winnicott (1992b) le da suma importancia a la creatividad y dice que el sentido de esta no debe limitarse a producciones exitosas y reconocidas por los demás, sino a una actitud frente a la vida que da color a la experiencia y una nueva visión sobre la realidad externa. Para la creatividad, nos dice, se necesita sentir que uno existe. La creatividad es el hacer que surge del ser. Creo que se puede decir que Winnicott hace de la creatividad un concepto central en su obra al punto de entender el desarrollo 
emocional saludable como la posibilidad de transitar creativamente por el mundo. La existencia de un self verdadero es la base de la creatividad. El self falso produce más bien una sensación de irrealidad o un sentimiento de futilidad. El self verdadero emana de la vida de la que están dotados los tejidos del cuerpo y de la acción de las funciones corporales. Se halla estrechamente ligado a la idea de un proceso primario y al principio no es reactivo a los estímulos externos. El bebé nace con un potencial heredado que -si todo marcha bien, si no sucede algo catastrófico en el ambiente que lo sostiene- se desarrollará en el sentido de la salud y la maduración.

En la estructuración del self se da la dependencia y su evolución hacia la independencia (Winnicott, 1992a, 1993). El niño se encuentra en un estado de dependencia absoluta. El bebé depende pero no tiene idea de su dependencia y por lo tanto reina la omnipotencia y el narcisismo primario. Gradualmente el niño tendrá un periodo de dependencia relativa, caracterizado por la separación entre él y el ambiente (madre), donde el niño se da cuenta de que sus suministros para sobrevivir provienen de otro, exterior a él. Si todo va bien, el niño se desarrollará hacia la independencia. La independencia absoluta no existe, ya que el ser humano saludable siempre es social, no aislado, lo cual implica siempre un cierto grado de dependencia.
La integración es un proceso que depende de una tendencia psicológica heredada, y que debe ser apoyada o complementada por una actividad del ambiente materno a la que Winnicott denomina holding o sostenimiento (Winnicott, 1992a). Al nacer el bebé cuenta con elementos motores y sensoriales rudimentarios que le darán una pauta de existencia, serán la matriz conductora de una experiencia de continuidad existencial, sobre la cual se asentarán los rudimentos de una elaboración imaginativa del funcionamiento corporal. En los comienzos se inicia un desarrollo desde un estado no integrado que resulta no traumático porque la madre conjura con su adaptación la angustia que suscitaría el derrumbe, si ella fallara. La protección que ofrece la madre al yo incipiente del bebé le evita la angustia inconcebible, que tiene distintos modos de ser vivenciada, todos los cuales se vinculan con las angustias psicóticas: fragmentarse, caer interminablemente, desvincularse del cuerpo. La aparición de esta angustia en cualquiera de sus formas provoca la ruptura de la continuidad existencial. Si perdura en el tiempo o es reiterada puede causar diversas patologías. Winnicott diferencia la desintegración (proceso defensivo generador de patologías) de la no integración (estado natural del cual se parte para llegar a la integración gracias al adecuado sostenimiento materno) a la cual se puede regresar en ciertos momentos, destacando que a es- 
te estado regresamos cada vez que somos capaces de recogernos sobre nosotros mismos antes de realizar un acto creativo.

El holding de la madre tiene que ver con su capacidad de empatía con las necesidades del bebé en el momento de la dependencia absoluta (es decir cuando aún no se ha producido la separación psicológica entre el yo y el no-yo). Esto incluye el sostenimiento físico del niño, la satisfacción de las necesidades fisiológicas y la protección frente a los estímulos displacenteros en una rutina del cuidado a lo largo del día y la noche. En realidad, nos habla de holding en general, pero este puede ser visto como concreto o metafórico; el concreto tendrá que ver con los cuidados rutinarios del bebé, y será más específicamente denominado handling o manipulación. El término holding quizá deba dejarse para su sentido de sostenimiento psicológico. El holding tiene que ver con la transmisión del ser psicológico, y el handling hará posible la experiencia de ser uno con el propio cuerpo.

Esto nos lleva al concepto de personalización, donde los cuidados regulares permiten el desarrollo de la unidad psicosomática (Panceira, 1997). La personalización es un momento de la integración que se refiere específicamente a la integración psicosomática y se produce concomitantemente con la etapa de diferenciación yo no-yo, paralelo al establecimiento de la piel como límite entre el adentro y el afuera, entre el bebé y la madre. Se va completando el esquema corporal del infante, y comienzan a tener sentido los términos introyección y proyección. La contrapartida facilitadora de este fenómeno - como ya he adelantado- es la manipulación o handling, que supone que la persona que cuida al niño es capaz de conducir al bebé y al cuerpo de este como si los dos formaran una unidad. Sus consecuencias serán una buena coordinación motora $\mathrm{y}$ un adecuado tono muscular.

Winnicott (1979) asume que la vida no deriva del instinto y que hay un estado anterior al sentirse vivo en el cual no hay aún vitalidad; de allí emana el ser. La madre deja surgir el ser del hijo siendo y no haciendo. Pero desde el comienzo hay experiencias paroxísticas suscitadas por la tensión generada por los instintos, una movilidad primaria, una vida de la que están dotados los tejidos. La energía primitiva es una energía no diferenciada y en el amor primitivo interviene un componente agresivo no intencional. Gradualmente se van diferenciando las pulsiones y la libido encuentra una satisfacción plena en la experiencia de la mamada, en relación con un objeto subjetivo, creado por el niño.

Hemos dicho que el rol materno de sostenimiento acompaña la integración del yo y permite el pasaje de la dependencia a la independencia. La experiencia de ilusión-desilusión llevará a 
la constitución del objeto transicional (Winnicott, 1990). En esta alternancia de ilusión-desilusión el bebé crea un puente imaginario que le permite mantener la integridad del yo y la continuidad existencial, a la vez que la ilusión del reencuentro con la madre. La evoca a partir de las huellas de la percepción, de un modo cercano a lo alucinatorio, que representa el inicio de los procesos transicionales. Estas experiencias son las precursoras de la capacidad para el uso de símbolos y de la apertura hacia los fenómenos culturales.

Winnicott (1990) observó que los bebés utilizan algunos objetos, reales y concretos, con los que establecen una relación impregnada de subjetividad. Estos serían los objetos transicionales, los cuales ayudan a desarrollar la capacidad de espera y a tolerar la frustración. El objeto ocupa el lugar de la madre, pero no la reemplaza totalmente; más bien elabora la ausencia. Hay un registro en el niño de que este objeto lo acompaña en ausencia de su madre, pero no es la madre. Representarla implica el reconocimiento de la pérdida y su recuperación en la fantasía. Este es el modelo de lo que serán todos los procesos de simbolización. Con el tiempo, gradualmente, los objetos transicionales van perdiendo su significación. La transicionalidad no es un fenómeno evolutivo propio de una etapa sino un modo de funcionamiento psíquico que luego será trasladado a otras experiencias, pasando de un objeto ele- gido a la multiplicidad de objetos abstractos y variables, permitiendo así el acceso a la cultura.

Esta relación con las primeras posesiones se realiza en una zona intermedia entre la realidad psíquica y la realidad externa, zona entre el yo y el noyo. Esta área es el espacio transicional donde se despliegan los fenómenos transicionales. Esta teorización permite registrar el pasaje de los estados subjetivos al reconocimiento de la exterioridad. La crianza de los niños debe favorecer el despliegue de la transicionalidad, ya que esta está vinculada al aprendizaje creador, al arte; y más bien la inhibición de la originalidad y los prejuicios están vinculados con el fracaso en la construcción de los fenómenos transicionales. Estos fenómenos son entendidos como universales. Se trata de un modo de uso de una actividad mental relacionada con la fantasía, que ocupa el espacio intermedio entre el mundo interno y el externo y que le permite al infante efectuar la transición necesaria entre la primera relación oral con la madre y una verdadera relación objetal.

La posición depresiva es un logro evolutivo que conduce a la adquisición del sentimiento de culpa e implica retener una angustia y una esperanza durante un periodo de tiempo, para lo cual se necesita que el bebé se perciba ya como persona completa capaz de relacionarse con otra persona completa. El niño descarga tensiones instinti- 
vas que son satisfactorias porque son su medio de expresión, pero al mirar atrás querrá reparar. Hay una descarga instintiva y la madre sostiene la situación; el niño percibe que la madre, a pesar de haber participado de la experiencia, la ha sobrevivido y está tranquila. Si esto sucede reiteradamente, a la larga el niño notará la diferencia entre la realidad y la fantasía, entre la realidad externa y la interna. El logro se da gradualmente entre los cinco y doce meses, coincidentes con el tiempo natural del destete y su instauración no es necesariamente definitiva hasta una fecha muy posterior.

A partir del sentimiento de culpa, se inicia un desarrollo que no concluye nunca y que atraviesa sucesivas etapas, que nace de la percepción del dolor que se siente como consecuencia del dolor que se ha causado real o imaginativamente a otro, sobre todo cuando posteriormente interviene la intención agresiva hacia otro. Este sentimiento se va haciendo más tolerable en la medida en que se puede hacer algo respecto al mal causado: si el objeto sobrevive, no registra cambios ni deseos de venganza y da lugar a la reparación a través de una sonrisa o un gesto cualquiera. La preocupación por el otro se refiere al hecho de que el individuo se interesa, le importa el otro y acepta la responsabilidad sobre sus ideas y comportamientos. Si esto no se produce, no se instaura la posibilidad de preocuparse por el otro, con la consecuente dificultad en la elaboración o expresión de la agresión. Solo pueden expresar su agresividad aquellas personas que sientan confianza en su posibilidad de reparación. El objeto debe sobrevivir sin vengarse.

Hasta aquí lo que extraigo como esencial de la teoría winnicottiana sobre el desarrollo emocional en el infante. Quisiera ahora reflexionar y especular un poco sobre lo que considero el desarrollo emocional de la madre en el proceso de maternidad, durante la temprana infancia del bebé.

Para la madre, creo, el principio está en donde empieza el yo del bebé, en su fantasía, es decir, en el deseo de ser madre. Este deseo puede ser consciente o no, lo cual no siempre resulta tan claro. Por ejemplo, Pines (1972) diferencia entre deseo de quedar embarazada y el deseo de convertirse en madre. Raphael-Leff (1995) nos recuerda que cada embarazo es diferente en tanto cada madre tendrá sus propios sentimientos, temores, esperanzas, recuerdos y poderosas mitologías inconscientes, de modo que un bebé imaginario se yuxtapone al embrión implantado en su vientre. Entonces, la madre surge en la fantasía mucho antes que el bebé. Quizá incluso antes de la posibilidad de reproducción. Sin embargo, en algún momento, lo psíquico se expresa en lo biológico, consciente o inconscientemente, y una mujer permite que un nuevo ser se albergue en ella. El mismo Winnicott (1992a) planteaba que el replegamiento de la madre está relacio- 
nado con el deseo de tener un hijo y con la posibilidad de identificarse con él, aún antes de su nacimiento.

Sin embargo, la tendencia a reprimir esta vivencia de replegamiento es, en mi opinión, bastante relativa. Creo que, en general, las madres recuerdan con mucha claridad este estado, aunque algunas tal vez quisieran olvidarlo. En todo caso, se trataría de reprimir solo ciertos aspectos de este replegamiento, quizá los más primarios, como expondré más adelante. Creo también que esto está en la base de la decisión de muchas madres de no tener más que un solo hijo. Si bien pueden existir muchas racionalizaciones de todo tipo para ello, me he encontrado con mujeres que tienen temor a un nuevo embarazo y a un nuevo nacimiento porque ese estado de replegamiento les resulta difícil de manejar en tanto la regresión que implica les trae dificultades en su entorno, cuando este no sostiene adecuadamente. Es posible también que la tendencia en nuestra cultura sea a reprimir cada vez menos esta vivencia, gracias a la difusión de teorías como la de Winnicott, que ponen de relieve lo natural de esta fase.

En relación con el objeto hallado y creado a la vez, pensemos en madres que no han creado al objeto, solo lo han hallado. Y esto puede tener muchas lecturas: un embarazo no deseado; un deseo de embarazo que se confunde y se convierte en maternidad no deseada; o una sobreimposición del medio. Win- nicott planteó que el medio debe posibilitar -y jamás modelar- para evitar un desarrollo patológico y reactivo fruto de la intrusión del medio en el área propia del sujeto. Creo que esto es cierto también para la madre: el entorno presiona con sus expectativas y existe, lamentablemente, mucha hipocresía al respecto. No solo se espera que a cierta edad una mujer se convierta en madre, sino que también se espera que la madre sea el prototipo de amor y madurez (aún en el quirófano). Se idealiza la maternidad, se da por sentado que el día del nacimiento de un hijo es el día más feliz en la vida de una mujer (muchas madres lo declaran así públicamente), y se rechaza toda manifestación contraria a esto. Lo cierto es que cualquier parturienta -sobre todo si es primeriza-, antes que felicidad, sentirá angustia (Lester y Malkah, 1986). Y mucho desconcierto. Un desconcierto físico y psíquico. Físico porque el dolor intenso se transforma de un instante a otro en ningún dolor, alternadamente; y en el nivel psíquico, se desconcierta porque no siente lo que el medio le exige que sienta. Se desconcierta de sí misma, se cuestiona si todo está bien con ella, si es una madre 'desnaturalizada'. En los primeros instantes después del parto, probablemente lo más cercano que puede sentirse al amor es curiosidad. Y no puede reconocer ante otros cómo se siente; a veces, tampoco ante sí misma. Del mismo modo en que Winnicott (Abadi, 1997; 
Hughes, 1990) habla de pacientes que arman la realidad desde afuera hacia adentro (personas que de los datos exteriores deducen cómo deben estarse sintiendo), la madre - a partir de las imágenes que ha interiorizado en su socialización- supone que debe estar amando a su hijo, y con esto se le priva de observar uno de los misterios más grandes de su ser: su propia capacidad de desarrollar amor. Esta, creo, es la primera desviación de la oportunidad que su experiencia de maternidad le ofrece para encontrarse más auténticamente. Pero el amor aparecerá, por supuesto; demorará quizá unas horas, o unos días. Y no es gratuito. La sensación es que el bebé se lo ha ido ganando; y se lo ha ganado, parafraseando a Winnicott, simplemente con ser.

Ahora, volviendo al replegamiento, el nacimiento es un momento crucial, donde este replegamiento se extrema. Y la regresión se extrema también -sobre todo para una primeriza- favorecida por la angustia de lo desconocido -sin importar cuántas historias haya escuchado-, y por la anticipación a un dolor mitificado. Winnicott (Abadi, 1996) decía que la vida es un acto simple de organización que va del centro a la periferia. Hay un ser esencial que existe y que va saliendo, va manifestándose hacia fuera. El dolor del primer alumbramiento es de una naturaleza muy particular. Es un dolor inimaginable y, me atrevo a decir, que no tanto por su intensidad-que puede ser muy subjetiva- sino por su naturaleza y por su localización. Es un dolor que se ubica en el centro mismo del cuerpo. Y de ese centro se irradia al resto del cuerpo. Repentinamente, un término tan primario como 'entrañas' cobra significación. Esa conciencia corporal, ese descubrimiento de dónde está el centro físico, un centro que se siente en ese momento como regidor de todo el resto de sensaciones, está indisolublemente ligado a una vivencia de centro psíquico. El repentino darse cuenta de estar creando una vida, y que al hacerlo uno puede estar a la vez muy cerca de la muerte, es una experiencia desorganizante que acaba de pronto con un suceso -doloroso- que le da forma a todo. En mi opinión, esto es lo que tiende a reprimirse, en mayor medida que el replegamiento en sí; esta regresión a una fase tan primaria e indiferenciada, en donde todo surge del cuerpo, en donde uno es todo y es nada. Y en donde uno es un dios creador. Para un adulto, una vivencia así puede llegar a ser aterradora.

Como ya he planteado, la sensación de no-integración que Winnicott reconoce en el principio de la vida es diferente a la desintegración, que sería un proceso defensivo generador de patologías. Para él la no-integración es un estado natural, inicial, del cual se parte para llegar a la integración, y al cual se puede regresar en ciertos momentos, gracias al adecuado sostenimiento materno, para crear. Desde esta perspecti- 
va, un alumbramiento es necesariamente un momento de no-integración psíquica.

Volviendo a la omnipotencia, podemos decir también que en los casos de embarazos deseados, la mujer que ha fantaseado un bebé se identifica con él aún antes de su concepción, y se lo ofrece a sí misma, se lo presenta a sí misma como objeto en el momento preciso de su propia necesidad (reloj biológico, contexto de su vida actual, etcétera). Lo crea. Pero este bebé que ya es, irá siendo, y con ello le irá haciendo saber que las necesidades de ambos no siempre coinciden, la irá desilusionando gradualmente, cada vez que el pecho de ella esté lleno y él solo quiera dormir; cada vez que ella quiera dormir y él quiera pecho; cada vez que ella quiera abrazarlo y él tenga calor. Y así poco a poco la volverá a la realidad, la realidad que ella ha abandonado parcialmente para agotarse en la vivencia con él. Realidad que será ya plenamente evidente mucho después, por ejemplo el primer día del nido, cuando la madre llega con dudas a recogerlo para encontrarlo feliz con sus nuevos amigos. Porque tenemos que decir que el ambiente de la madre también es el bebé, y si bien reconoce que este no agota la realidad, metafóricamente sí lo hace durante un tiempo, y ella se siente extraña fuera de este ambiente. Lejos de su bebé, bajo el pretexto o la ilusión de que él la necesita y que debe volver con él, estoy convencida de que está la razón más antigua de su propia historia, la cual está reeditando: ella necesita estar cerca del otro polo de su unidad, su bebé.

Y pensando en los objetos transicionales que tiene la madre, que representan a su bebé, podemos mencionar muchos: un mechón de pelo, el primer diente, su primer zapatito, solo por citar algunos. Objetos subjetivizados, que muchas veces carga consigo, en una cadena, o en la cartera, que hacen más llevaderas las ausencias, reconociéndolas a la vez que negándolas, y anunciando el reencuentro.

Retomando la idea de la capacidad de preocuparse por el otro, parecería que esta ya está dada antes de que el bebé nazca; es más, para Winnicott, en el nivel genital del desarrollo la preocupación por el otro está en la base de la familia cuando los progenitores, más allá del placer de su relación sexual, asumen la responsabilidad por el resultado de esta. Sin embargo, con el nacimiento de un hijo, esta capacidad también se reedita. Y la posibilidad de una culpa auténtica, así como de reparar. En el caso de la madre, creo, esto va muy ligado a su propio proceso de separación-individuación. Curiosamente, y no por casualidad, esta fase de individuación empieza aproximadamente cuando llega la época normal del destete. En la medida en que el niño tiene otras necesidades nutricionales, él mismo va dejando el pecho, y esto ocurre cuando también tiene otras necesidades 
psíquicas, como por ejemplo, incluir a otros en lo que considera su 'ambiente'. Con el destete la madre también se independiza físicamente, y se permite mayores alejamientos, por más tiempo. Y resulta importante para la madre comprobar que, a pesar de no ser el pecho, aún es especial. Al alejarse con más frecuencia la madre teme, por ejemplo, estar siendo egoísta. O se avergüenza de su deseo y necesidad de separarse y 'descansar'. Teme dañar al bebé, que ya claramente reacciona ante la separación. Pero se tranquiliza al regresar y encontrar no solo que ha sobrevivido, sino que su amor ha sobrevivido, pues se alegra de verla, y le permite reparar: él permite que ella lo bese, lo abrace, y disfrutan ambos del reencuentro.

Para concluir diremos que en la vida personal que nace de uno mismo, no del otro, y que lleva al otro naturalmente, hay un centro personal que organiza todas las identificaciones. La madre brinda un sostén que permite la integración temporal y espacial, sin que el bebé lo sepa. Pero el bebé también lo hace con la madre, sin que ella lo sepa. A través de la identificación con su niño, la madre tiene una nueva oportunidad de mejorar integraciones, de completar círculos identificatorios, de maduración emocional. Puede o no utilizar esta oportunidad para hacerlo; puede, también, en el peor de los casos, utilizar la identificación con su bebé para llenar -sin elaboración- vacíos no resueltos en su historia. Pero si la capacidad de estar solo depende de la existencia de un objeto suficientemente bueno en la realidad psíquica del sujeto, si esta capacidad implica que el sujeto ha tenido la oportunidad, gracias a una buena maternalización, de formarse poco a poco la creencia en un medio ambiente benigno, podemos afirmar también que no hay nada en el mundo que posibilite esta confianza en la bondad del medio, como la experiencia de observar el surgimiento de un ser nuevo, y la propia contribución a ese surgimiento.

\section{REFERENCIAS}

Abadi, S. (1996). Transiciones. El modelo terapéutico de D. W. Winnicott. Buenos Aires: Lumen.

Abadi, S. (1997). Desarrollos posfreudianos: Escuelas y autores. Buenos Aires: Editorial Belgrano.

Bibring, G. (1961). A study of the psychological process in pregnancy and of the earliest mother-child relationship. Psychoanalytical Study of the Child, 16, 9-44.

Hughes, J. (1990). Reshaping the psychoanalytic domain. The work of Melanie Klein, $W$. R. D. Fairbairn y D. W. Winnicott. Los Angeles: University of California Press.

Lester, E. \& Malkah, N. (1986). Crisis evolutiva y relaciones objetales en el embarazo: Una consideración psicoanalítica. International Journal of Psychoanalysis, 67, 357. 
Panceira, A. (1997). Clínica psicoanalítica a partir de la obra de Winnicott. Buenos Aires: Lumen.

Pines, D. (1972). Pregnancy and motherhood: interaction between fantasy and reality. British Journal of Medical Psychology, 45, 333-343.

Raphael-Leff, J. (1995). Pregnancy, the inside story. Nueva Jersey: Jason Aronson Inc.

Winnicott, D. W. (1979). El proceso de maduración en el niño. Barcelona: Laia.
Winnicott, D. W. (1990). Objetos transicionales y fenómenos transicionales. Barcelona: Laia.

Winnicott, D. W. (1992a). Los procesos de maduración y el ambiente facilitador. Barcelona: Gedisa.

Winnicott, D. W. (1992b). Realidad y juego. Barcelona: Gedisa.

Winnicott, D. W. (1993). El niño y el mundo externo. Buenos Aires: Hormé. 